\title{
Slip Effects on Peristaltic Transport of a Particle-Fluid Suspension in a Planar Channel
}

\author{
Mohammed H. Kamel, ${ }^{1}$ Islam M. Eldesoky, ${ }^{2}$ Bilal M. Maher, ${ }^{2}$ and Ramzy M. Abumandour ${ }^{2}$ \\ ${ }^{1}$ Department of Engineering Mathematics and Physics, Faculty of Engineering, Cairo University, Giza, Egypt \\ ${ }^{2}$ Department of Basic Engineering Sciences, Faculty of Engineering, Menoufia University, Shebin El-Kom, Egypt \\ Correspondence should be addressed to Islam M. Eldesoky; eldesokyi@yahoo.com
}

Received 1 August 2014; Revised 16 March 2015; Accepted 16 April 2015

Academic Editor: Agnès Drochon

Copyright (C) 2015 Mohammed H. Kamel et al. This is an open access article distributed under the Creative Commons Attribution License, which permits unrestricted use, distribution, and reproduction in any medium, provided the original work is properly cited.

Peristaltic pumping induced by a sinusoidal traveling wave in the walls of a two-dimensional channel filled with a viscous incompressible fluid mixed with rigid spherical particles is investigated theoretically taking the slip effect on the wall into account. A perturbation solution is obtained which satisfies the momentum equations for the case in which amplitude ratio (wave amplitude/channel half width) is small. The analysis has been carried out by duly accounting for the nonlinear convective acceleration terms and the slip condition for the fluid part on the wavy wall. The governing equations are developed up to the second order of the amplitude ratio. The zeroth-order terms yield the Poiseuille flow and the first-order terms give the Orr-Sommerfeld equation. The results show that the slip conditions have significant effect within certain range of concentration. The phenomenon of reflux (the mean flow reversal) is discussed under slip conditions. It is found that the critical reflux pressure is lower for the particle-fluid suspension than for the particle-free fluid and is affected by slip condition. A motivation of the present analysis has been the hope that such theory of two-phase flow process under slip condition is very useful in understanding the role of peristaltic muscular contraction in transporting biofluid behaving like a particle-fluid mixture. Also the theory is important to the engineering applications of pumping solid-fluid mixture by peristalsis.

\section{Introduction}

Peristalsis is a form of a fluid transport induced by a progressive wave of area contraction or expansion along the walls of a distensible duct containing liquid. In physiology, peristaltic mechanism is involved in many biological organs such as ureter, gastrointestinal tract, ducts afferents of the male reproductive tracts, cervical canal, female fallopian tube, lymphatic vessels, and small blood vessels. In addition, peristaltic pumping occurring in many practical applications involving biomechanical systems such as roller, finger pumps, and heart-lung machine have been fabricated.

Since the first investigation of Latham [1], several theoretical and experimental studies have been conducted in the past to understand peristaltic action in different situations.

The literature on peristalsis is by now quite extensive, and a summary of most of the investigations has been presented in detail by Rath [2], L. M. Srivastava and V. P. Srivastava [35], Srivastava and Saxena [6], and V. P. Srivastava and L. M. Srivastava [7].
The theoretical study of the theory of particle-fluid mixture is very useful in understanding a number of diverse physical problems concerned with powder technology, fluidization, transportation of solid particles by a liquid, transportation liquid slurries in chemical and nuclear processing, and metalized liquid fuel slurries for rocketry. The sedimentation of particles in a liquid is of interest in much chemical engineering process, in medicine, where erythrocyte sedimentation has become a standard clinical test, and in oceanography as well as other fields. The particulate theory of blood has recently become the object of scientific research, Hill and Bedford [8], L. M. Srivastava and V. P. Srivastava [3-5, 9], Trowbridge [10], and Oka [11]. A number of research works on the topic, with and without peristalsis, have been reviewed by L. M. Srivastava and V. P. Srivastava [5]. Applications of the theory of particle-fluid mixture to the microcirculation and erythrocyte sedimentation included the work of Wang and Skalak [12], Bungay and Brenner [13], Skalak et al. [14], and Karino et al. [15]. 

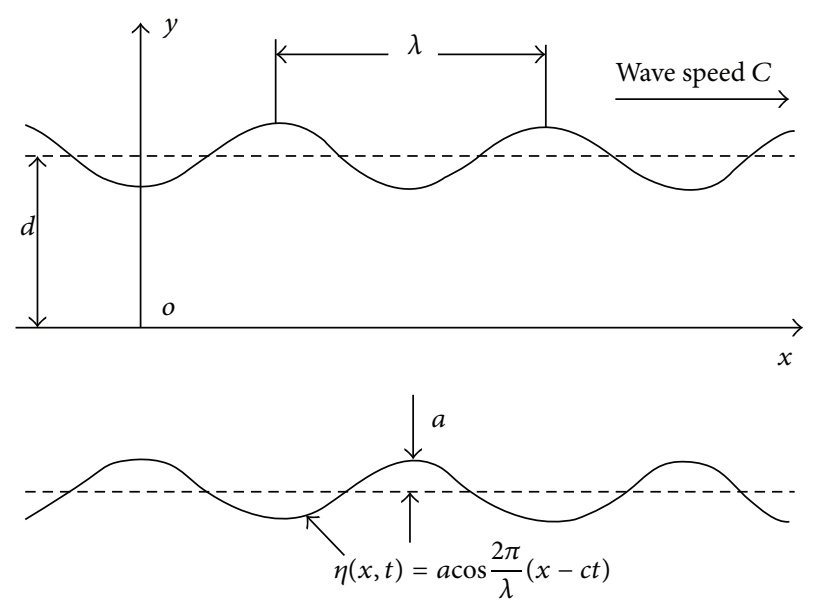

FIGURE 1: Geometry of the problem.

Peristaltic transport of solid particle with fluid was first attempted by Hung and Brown [16]. They initiated the study of the peristaltic transport of solid particles, which included an experimental work on the particle transport in twodimensional vertical channels having various geometries. In this connection also another paper by Brown and Hung [17] and a study by Takabatake and Ayukawa [18] are worth mentioning. Both studies have employed finite difference technique to solve two-dimensional nonlinear peristaltic flows problem. L. M. Srivastava and V. P. Srivastava [5] studied the peristaltic pumping of a particle-fluid mixture in a two-dimensional channel carried out mathematically; a perturbation solution is obtained. Mekheimer et al. [19] studied the peristaltic pumping of a particle-fluid suspension in a planar channel. El Misery et al. [20] studied the peristaltic motion of an incompressible generalized Newtonian fluid in a planar channel.

No-slip boundary conditions are convenient idealization of the behavior of viscous fluids near walls. The inadequacy of the no-slip condition is quite evident in polymer melts which often exhibit microscopic wall slip. The slip condition plays an important role in shear skin, spurt, and hysteresis effects. The boundary conditions relevant to flowing fluids are very important in predicting fluid flows in many applications. The fluids that exhibit boundary slip have important technological applications such as in polishing valves of artificial heart and internal cavities [21]. The slip effects on the peristaltic flow of a non-Newtonian Maxwellian fluid have been investigated by Eldesoky [22]. The influence of slip condition on peristaltic transport of a compressible Maxwell fluid through porous medium in a tube has been studied by Chu and Fang [23]. Many recent researches have been made in the subject of slip boundary conditions [24-33].

From the previous studies, there is no any attempt to study the effect of slip condition on the flow of a particlefluid suspension with peristalsis. The purpose of this paper is to study the slip effects on the peristaltic pumping of a particle-fluid mixture in a two-dimensional channel. It is an application of the two-dimensional analysis of peristaltic motion of a particle-fluid mixture by L. M. Srivastava and V. P. Srivastava [5] and the two-dimensional analysis of peristaltic motion of single phase fluid by Fung and Yih [34] in the presence of slip effect. The mathematical model considers a particle-fluid mixture between infinite parallel walls with slip condition on which a sinusoidal traveling wave is imposed. A perturbation solution is obtained which satisfies the momentum equations for the case in which amplitude ratio (wave amplitude/channel half width) is small. Finally, the phenomenon of the mean flow reversal is presented and its physiological implication is discussed. Beside the engineering applications of pumping particle-fluid mixture by peristalsis, the present analysis of two-phase flow process is potentially important in regard to biofluid transport by peristalsis muscular contractions in body organs where fluids behave like particle-fluid mixtures, namely, chime in small intestine, spermatic fluid in cervical canal, urine (from a diseased kidney) in ureter, and blood suspension in arteriole.

\section{Formulation of the Problem}

Consider a two-dimensional infinite channel of mean width $2 d$ (see Figure 1), filled with a mixture of small spherical rigid particles in an incompressible Newtonian viscous fluid. The walls of the channel are flexible, on which are imposed travelling, sinusoidal wave of small amplitude. The equations governing conservation of mass and linear momentum for both fluid and particle phase using a continuum approach are expressed as follows (Drew [35]; L. M. Srivastava and V. P. Srivastava $[5,9])$.

Fluid Phase

$$
\begin{aligned}
(1-C) \rho_{f}\left[\frac{\partial u_{f}}{\partial t}+u_{f} \frac{\partial u_{f}}{\partial x}+v_{f} \frac{\partial u_{f}}{\partial y}\right] \\
=-(1-C) \frac{\partial p}{\partial x}+(1-C) \mu_{s}(C) \nabla^{2} u_{f} \\
+C S\left(u_{p}-u_{f}\right)
\end{aligned}
$$




$$
\begin{gathered}
(1-C) \rho_{f}\left[\frac{\partial v_{f}}{\partial t}+u_{f} \frac{\partial v_{f}}{\partial x}+v_{f} \frac{\partial v_{f}}{\partial y}\right] \\
=-(1-C) \frac{\partial p}{\partial y}+(1-C) \mu_{s}(C) \nabla^{2} v_{f} \\
+C S\left(v_{p}-v_{f}\right), \\
\frac{\partial}{\partial x}\left\{(1-C) u_{f}\right\}+\frac{\partial}{\partial y}\left\{(1-C) v_{f}\right\}=0 .
\end{gathered}
$$

Particulate Phase

$$
\begin{gathered}
C \rho_{p}\left[\frac{\partial u_{p}}{\partial t}+u_{p} \frac{\partial u_{p}}{\partial x}+v_{p} \frac{\partial u_{p}}{\partial y}\right] \\
=-C \frac{\partial p}{\partial x}+C S\left(u_{f}-u_{p}\right), \\
C \rho_{p}\left[\frac{\partial v_{p}}{\partial t}+u_{p} \frac{\partial v_{p}}{\partial x}+v_{p} \frac{\partial v_{p}}{\partial y}\right] \\
=-C \frac{\partial p}{\partial y}+C S\left(v_{f}-v_{p}\right), \\
\frac{\partial}{\partial x}\left\{C u_{p}\right\}+\frac{\partial}{\partial y}\left\{C v_{p}\right\}=0 .
\end{gathered}
$$

In (1)-(6), $x$ and $y$ are Cartesian coordinates with $x$ measured in the direction of wave propagation and $y$ measured in the direction normal to the mean position of the channel walls, $\left(u_{f}, v_{f}\right)$ denotes fluid phase velocities, $\left(u_{p}, v_{p}\right)$ denotes particulate phase velocities, $\rho_{f}$ and $\rho_{p}$ are the actual densities of the materials constituting fluid and particulate phase, respectively, $(1-C) \rho_{f}$ is the fluid phase density, $C \rho_{p}$ is the particulate phase density, $p$ denotes the pressure, $C$ denotes the volume fraction density of the particles, $\mu(C)$ is the particle-fluid mixture viscosity (also the effective viscosity of the suspension), and $S$ the drag coefficient of interaction for the force exerted by one phase on the other.

The concentration of the particles is considered to be so small that the field interaction between particles may be neglected. Thus, the diffusivity terms, which can model the effects of particle-particle impacts due to the Brownian motion, are neglected. It is worth mentioning here that the effect of Brownian motion was considered by others including Batchelor [36, 37]. The volume fraction density, $C$, of the particles is chosen also constant. This is a good assumption for low concentration of small particles.

The expression for the drag coefficient for the present problem is selected as

$$
\begin{aligned}
S & =\frac{9}{2} \frac{\mu_{0}}{a^{2}} \lambda^{\prime}(C), \\
\lambda^{\prime}(C) & =\frac{4+3\left[8 C-3 C^{2}\right]^{1 / 2}+3 C}{[2-3 C]^{2}},
\end{aligned}
$$

where $\mu_{0}$ is the fluid viscosity and $a$ is the radius of the particle. Relation (7) represents the classical Stokes' drag for small particle Reynolds number, modified to account for the finite particulate fractional volume through the function $\lambda^{\prime}(C)$, obtained by Tam [38].

Many empirical relations have been suggested to express the viscosity of the suspension as a function of particle concentration and viscosity of the suspending medium. Einstein was the first to obtain theoretically that the viscosity of the suspension $\mu_{S}$ was related to that of the suspending medium $\mu_{0}$ for spheres in suspension by $\mu_{0}=\mu_{S}(1-2.5 C)$. However, the Einstein formula expresses the viscosity of the suspension only when $C$ is less than 0.05 . As $C$ increases from 0.05 , the suspension viscosity varies from the Einstein equation. For the present problem, an empirical relation for the viscosity of the suspension is as follows:

$$
\begin{aligned}
\mu_{S}(C) & =\mu_{0} \frac{1}{1-q C}, \\
q & =0.07 \exp \left[2.49 C+\frac{1107}{T} \exp (-1.69 C)\right],
\end{aligned}
$$

where $T$ the absolute temperature $\left({ }^{\circ} \mathrm{K}\right)$, suggested by Charm and Kurland (1974) [39], is used. The viscosity of the suspension expressed by this formula is found to be reasonably accurate up to $C=0.6$. Charm and Kurland (1974) [39] tested (8a) and (8b) with a cone and plate viscometer and found it to be in agreement within ten percent in case of blood suspension.

The boundary conditions that must be satisfied by the fluid on the walls are the slip and impermeability conditions. The walls of the channel are assumed to be flexible but extensible with a travelling sinusoidal wave, and displacement in the channel walls is in transverse direction only. Hence, boundary conditions are

$$
\begin{aligned}
& u_{f}=\Psi_{f y}=\mp A \frac{\partial u_{f}}{\partial y} \\
& v_{f}=-\Psi_{f x}= \pm \frac{\partial \eta}{\partial t} \text { on } y= \pm d \pm \eta \\
& v_{p}=-\Psi_{p x}= \pm \frac{\partial \eta}{\partial t}
\end{aligned}
$$

where $\Psi$, the stream function, is such that

$$
\begin{aligned}
& u_{f}=\frac{\partial \Psi_{f}}{\partial y}, \\
& v_{f}=-\frac{\partial \Psi_{f}}{\partial x}, \\
& u_{p}=\frac{\partial \Psi_{p}}{\partial y}, \\
& v_{p}=-\frac{\partial \Psi_{p}}{\partial x} .
\end{aligned}
$$

The transverse displacement, $\eta$, of the wall is represented as

$$
\eta(x, t)=a \cos \frac{2 \pi}{\lambda}(x-c t),
$$


where $a$ is the amplitude, $\lambda$ the wavelength, and $c$ the wave speed.

We now select the following set of nondimensional variables and parameters:

$$
\begin{aligned}
x^{\prime} & =\frac{x}{d}, \\
y^{\prime} & =\frac{y}{d}, \\
u_{f}^{\prime} & =\frac{u_{f}}{c}, \\
v_{f}^{\prime} & =\frac{v_{f}}{c}, \\
u_{p}^{\prime} & =\frac{u_{p}}{c}, \\
v_{p}^{\prime} & =\frac{v_{p}}{c}, \\
\eta^{\prime} & =\frac{\eta}{d}, \\
\Psi_{f}^{\prime} & =\frac{\Psi_{f}}{c d}, \\
\Psi_{p}^{\prime} & =\frac{\Psi_{p}}{c d}, \\
t^{\prime} & =\frac{c t}{d}, \\
p^{\prime} & =\frac{p}{\rho_{f} c^{2}}, \\
\rho_{f} & \mu_{0}
\end{aligned}
$$

Suspension Reynolds number

$$
R=\frac{c d \rho_{f}}{(1-C) \mu_{S}} .
$$

Wave number

$$
\alpha=\frac{2 \pi d}{\lambda}
$$

Knudsen number

$$
\mathrm{Kn}=\frac{A}{R}
$$

Amplitude ratio

$$
\varepsilon=\frac{a}{d}
$$

Suspension parameter

$$
M=\frac{S d^{2}}{(1-C) \mu_{S}} .
$$

Suspension parameter

$$
N=\frac{S d^{2} \rho_{f}}{(1-C) \rho_{p} \mu_{S}} .
$$

Thus, the systems of (1)-(6), and (9a)-(11) now become, after dropping the primes,

$$
\begin{aligned}
& (1-C) R\left[\frac{\partial}{\partial t} \nabla^{2} \Psi_{f}+\Psi_{f y} \nabla^{2} \Psi_{f x}-\Psi_{f x} \nabla^{2} \Psi_{f y}\right] \\
& =\nabla^{4} \Psi_{f}+C M\left(\nabla^{2} \Psi_{p}-\nabla^{2} \Psi_{f}\right), \\
& C R\left[\frac{\partial}{\partial t} \nabla^{2} \Psi_{p}+\Psi_{p y} \nabla^{2} \Psi_{p x}-\Psi_{p x} \nabla^{2} \Psi_{p y}\right] \\
& =C N\left(\nabla^{2} \Psi_{f}-\nabla^{2} \Psi_{p}\right), \\
& \eta=\varepsilon \cos \alpha(x-t), \\
& \Psi_{f y}=\mp \operatorname{Kn} \Psi_{f y y}, \\
& \Psi_{f x}=\mp \alpha \varepsilon \sin \alpha(x-t), \\
& \Psi_{p x}=\mp \alpha \varepsilon \sin \alpha(x-t),
\end{aligned}
$$

$$
\text { on } y= \pm(1+\eta) \text {, }
$$

where $\nabla^{2}$ denotes the Laplacian operator.

\section{Method of Solution}

Assuming the amplitude ratio $e$ of the wave is small, we obtain the solution for the stream function as a power series in terms of $\varepsilon$, by expanding $\Psi_{f}, \Psi_{p}$, and $\partial p / \partial x$ in the form (Fung and Yih (1968) [34])

$$
\begin{aligned}
& \Psi_{f}=\Psi_{f 0}+\varepsilon \Psi_{f 1}+\varepsilon^{2} \Psi_{f 2}+\cdots \\
& \Psi_{p}=\Psi_{p 0}+\varepsilon \Psi_{p 1}+\varepsilon^{2} \Psi_{p 2}+\cdots \\
& \frac{\partial p}{\partial x}=\left(\frac{\partial p}{\partial x}\right)_{0}+\varepsilon\left(\frac{\partial p}{\partial x}\right)_{1}+\varepsilon^{2}\left(\frac{\partial p}{\partial x}\right)_{2}+\cdots
\end{aligned}
$$

In (25), the first term on the right-hand side corresponds to the imposed pressure gradient associated with the primary flow and the other terms correspond to the peristaltic motion or higher imposed pressure gradient.

Substituting (23) and (24) in (19), (20), and (22) and collecting terms of like powers of $\varepsilon$, we obtain three sets of coupled linear differential equations with their corresponding boundary conditions in $\Psi_{(f, p) 0}, \Psi_{(f, p) 1}$, and $\Psi_{(f, p) 2}$, for the first three powers of $\varepsilon$.

The first set of differential equations in $\Psi_{(f, p) 0}$, subject to the steady parallel flow and transverse symmetry assumption for a constant pressure gradient in the $x$-direction, yields 
the following classical Poiseuille flow for the fluid and the particulate phase:

$$
\begin{aligned}
\Psi_{f 0} & =K\left[\left(y-\frac{1}{3} y^{3}\right)+\frac{2 \mathrm{Kn}}{2+\mathrm{Kn}}\left(y-\frac{1}{2} y^{2}\right)\right], \\
\Psi_{p 0} & =K\left(y+\frac{2 y}{M}-\frac{1}{3} y^{3}\right), \\
K & =-\frac{R}{2}\left(\frac{\partial p}{\partial x}\right)_{0},
\end{aligned}
$$

where $K$ is the Poiseuille flow parameter.

Thus, the effect of the particles on the fluid velocity profile is to cause an increase in the viscosity; that is, fluid viscosity $\mu_{0}$ is replaced by suspension viscosity (see (8a) and (8b)), and thus for a given pressure difference less fluid will flow through the channel. Further, the particles lead the fluid by a relative velocity proportional to $1 / M(\partial p / \partial x)_{0}$.

The second and third sets of differential equations in $\Psi_{(f, p) 1}$ and $\Psi_{(f, p) 2}$ with their corresponding boundary conditions are satisfied by

$$
\begin{aligned}
2 \Psi_{f 1}(x, y, t)= & \Phi_{f 1}(y) e^{i \alpha(x-t)}+\Phi_{f 1}^{*}(y) e^{-i \alpha(x-t)}, \\
2 \Psi_{p 1}(x, y, t)= & \Phi_{p 1}(y) e^{i \alpha(x-t)}+\Phi_{p 1}^{*}(y) e^{-i \alpha(x-t)}, \\
2 \Psi_{f 2}(x, y, t)= & \Phi_{f 20}+\Phi_{f 22}(y) e^{2 i \alpha(x-t)} \\
& +\Phi_{f 22}^{*}(y) e^{-2 i \alpha(x-t)} \\
2 \Psi_{p 2}(x, y, t)= & \Phi_{p 20}+\Phi_{p 22}(y) e^{2 i \alpha(x-t)} \\
& +\Phi_{p 22}^{*}(y) e^{-2 i \alpha(x-t)} .
\end{aligned}
$$

A substitution of (29a), (29b) and (30a), (30b) into the differential equations and their corresponding boundary conditions in $\Psi_{(f, p) 1}$ and $\Psi_{(f, p) 2}$ leads to the following set of differential equations:

$$
\begin{aligned}
& {\left[\frac{d^{2}}{d y^{2}}-\alpha^{2}+i(1-C)\right.} \\
& \left.\cdot \alpha R\left[1-K\left[\left(1-y^{2}\right)+\frac{2 \mathrm{Kn}}{2+\mathrm{Kn}}(1-y)\right]\right]\right] \\
& \cdot\left(\frac{d^{2}}{d y^{2}}-\alpha^{2}\right) \Phi_{f 1}-2 i K(1-C) \alpha R \Phi_{f 1} \\
& =C M\left(\frac{d^{2}}{d y^{2}}-\alpha^{2}\right)\left(\Phi_{f 1}-\Phi_{p 1}\right),
\end{aligned}
$$

$$
\begin{aligned}
& i C \alpha R[1 \\
& \left.-K\left[\left(1-y^{2}+\frac{2}{M}\right)+\frac{2 \mathrm{Kn}}{2+\mathrm{Kn}}\left(1-y+\frac{2}{M}\right)\right]\right] \\
& \cdot\left(\frac{d^{2}}{d y^{2}}-\alpha^{2}\right) \Phi_{p 1}-2 i K C \alpha R \Phi_{p 1}=C N\left(\frac{d^{2}}{d y^{2}}\right. \\
& \left.-\alpha^{2}\right)\left(\Phi_{p 1}-\Phi_{f 1}\right) \\
& \Phi_{f 1}^{\prime}( \pm 1)-2 K \mp \frac{2 K \mathrm{Kn}}{2+\mathrm{Kn}}=\mp \mathrm{Kn}\left[\Phi_{f 1}^{\prime \prime}( \pm 1) \mp 2 K\right], \\
& \Phi_{f 1}( \pm 1)= \pm 1 \\
& \Phi_{p 1}( \pm 1)= \pm 1 \text {, } \\
& \Phi_{f 20}^{\prime \prime \prime \prime}(y)=-\frac{(1-C) \alpha R}{2}\left(\Phi_{f 1} \Phi_{f 1}^{* \prime \prime}-\Phi_{f 1}^{*} \Phi_{f 1}^{\prime \prime}\right)^{\prime} \\
& +C M\left(\Phi_{f 20}^{\prime \prime}-\Phi_{p 20}^{\prime \prime}\right) \\
& 0=-\frac{i C \alpha R}{2}\left(\Phi_{p 1} \Phi_{p 1}^{* \prime \prime}-\Phi_{p 1}^{*} \Phi_{p 1}^{\prime \prime}\right)^{\prime}+C N\left(\Phi_{p 20}^{\prime \prime}\right. \\
& \left.-\Phi_{f 20}^{\prime \prime}\right) \\
& \left(\frac{d^{2}}{d y^{2}}-4 \alpha^{2}\right)\left[\frac{d^{2}}{d y^{2}}-4 \alpha^{2}+2 i(1-C) \alpha R\right] \Phi_{f 22} \\
& =2 i(1-C) K \alpha R\left[\left(1-y^{2}\right)+\frac{2 \mathrm{Kn}}{2+\mathrm{Kn}}(1-y)\right] \\
& \cdot\left(\frac{d^{2}}{d y^{2}}-4 \alpha^{2}\right) \Phi_{f 22}+4 i(1-C) K \alpha R \Phi_{f 22} \\
& +\frac{i(1-C) \alpha R}{2}\left(\Phi_{f 1}^{\prime} \Phi_{f 1}^{\prime \prime}-\Phi_{f 1} \Phi_{f 1}^{\prime \prime \prime}\right) \\
& +C M\left(\frac{d^{2}}{d y^{2}}-4 \alpha^{2}\right)\left(\Phi_{f 22}-\Phi_{p 22}\right), \\
& 2 i C \alpha R\left(\frac{d^{2}}{d y^{2}}-4 \alpha^{2}\right) \Phi_{p 22}=2 i C K \alpha R\left(1-y^{2}\right. \\
& \left.-\frac{2}{M}\right) \times\left(\frac{d^{2}}{d y^{2}}-4 \alpha^{2}\right) \Phi_{p 22}+4 i C K \alpha R \Phi_{p 22} \\
& +\frac{i C \alpha R}{2}\left(\Phi_{p 1}^{\prime} \Phi_{p 1}^{\prime \prime}-\Phi_{p 1} \Phi_{p 1}^{\prime \prime \prime}\right)+C N\left(\frac{d^{2}}{d y^{2}}\right. \\
& \left.-4 \alpha^{2}\right)\left(\Phi_{p 22}-\Phi_{f 22}\right) \\
& \Phi_{f 20}^{\prime}( \pm 1) \pm \frac{1}{2}\left[\Phi_{f 1}^{\prime \prime}( \pm 1)+\Phi_{f 1}^{* \prime \prime}( \pm 1)\right]-K \\
& =\mp \mathrm{Kn}\left[\Phi_{f 20}^{\prime \prime}( \pm 1) \pm \frac{1}{2}\left[\Phi_{f 1}^{\prime \prime \prime}( \pm 1)+\Phi_{f 1}^{* \prime \prime \prime}( \pm 1)\right]\right] \text {, }
\end{aligned}
$$




$$
\begin{aligned}
& \Phi_{f 22}^{\prime}( \pm 1) \pm \frac{1}{2} \Phi_{f 1}^{\prime \prime}( \pm 1)-\frac{K}{2}=\mp \mathrm{Kn}\left[\Phi_{f 22}^{\prime \prime}( \pm 1) \pm \frac{1}{2}\right. \\
& \left.\cdot \Phi_{f 1}^{\prime \prime \prime}( \pm 1)\right] \\
& \Phi_{f 22}( \pm 1) \pm \frac{1}{4} \Phi_{f 1}^{\prime}( \pm 1)=0, \\
& \Phi_{p 22}( \pm 1) \pm \frac{1}{4} \Phi_{p 1}^{\prime}( \pm 1)=0 .
\end{aligned}
$$

Thus, we obtained a set of differential equations together with the corresponding boundary conditions which are sufficient to determine the solution of the problem up to the second order in $\varepsilon$. Now, our main attention is to find out solution of differential equations for $\Phi_{f 1}$ and $\Phi_{p 1}$. Although (31) and (32) for $\Phi_{f 1}$ and $\Phi_{p 1}$ are coupled fourth-order ordinary differential equations with variable coefficients, it would, perhaps, be impossible to obtain solution of these differential equations for arbitrary values of $R, \alpha$, and $K$. This is just because of the moving boundary considered in the present problem. The condition of moving boundary has made the boundary condition nonhomogeneous and thus the problem is not an eigenvalue problem as in all problems of hydrodynamic stability for which solutions are available in the literature. However, we can restrict our investigation to the case of pumping of an initially stagnant fluid, corresponding to no imposed pressure gradient. Thus, in this case $(\partial p / \partial x)_{0}=0$, which means that constant $K$ vanishes and we would be able to obtain a simple closed form analytical solution of this interesting case of free pumping. Physically, this assumption means that the fluid is stationary if there are no peristaltic waves. In fact, this assumption is not so restrictive because the maximum pressure gradient that small-amplitude waves can generate is of the order of $\varepsilon^{2}$ and in the pumping range the zeroth-order mean pressure gradient must certainly vanish.

Solutions of (31) and (32) subject to the boundary condition ((33a), (33b), and (33c)), under the assumption, $K=0$, may be obtained as

$$
\begin{aligned}
& \Phi_{f 1}(y)=A_{1} \sinh (\alpha y)+B_{1} \sinh (\beta y), \\
& \Phi_{p 1}(y)=A_{2} \sinh (\alpha y)+B_{2} \sinh (\beta y),
\end{aligned}
$$

where

$$
\begin{aligned}
& \beta^{2}=\alpha^{2}-i \alpha R\left[1-C+\frac{C M}{N-i \alpha R}\right] \\
& A_{1}=\frac{-\left(\beta \cosh (\beta)+\mathrm{Kn} \beta^{2} \sinh (\beta)\right)}{\alpha \cosh (\alpha) \sinh (\beta)-\beta \cosh (\beta) \sinh (\alpha)+\mathrm{Kn} \sinh (\alpha) \sinh (\beta)\left(\alpha^{2}-\beta^{2}\right)}, \\
& B_{1}=\frac{\alpha \cosh (\alpha)+\mathrm{Kn} \alpha^{2} \sinh (\alpha)}{\alpha \cosh (\alpha) \sinh (\beta)-\beta \cosh (\beta) \sinh (\alpha)+\mathrm{Kn} \sinh (\alpha) \sinh (\beta)\left(\alpha^{2}-\beta^{2}\right)}, \\
& A_{2}=\frac{1-B_{2} \sinh (\beta)}{\sinh (\alpha)}, \\
& B_{2}=\frac{B_{1} N}{N-i \alpha R} .
\end{aligned}
$$

Next, in the expansion of $\Psi_{(f, p) 2}$, we need only to concern ourselves with the terms $\Phi_{(f, p) 20}(y)$ as our aim is to determine the mean flow only. Thus, the solution of the coupled differential equations ((34), (35)) subject to the boundary conditions ((38a), (38b), (38c), and (38d)), under the assumption, $K=0$, gives the expressions

$$
\begin{aligned}
\Phi_{f 20}^{\prime}(y)= & F(y)-F(1)+D-C_{1}\left(1-y^{2}+2 \mathrm{Kn}\right), \\
\Phi_{p 20}^{\prime}(y)= & G(y)-F(1)+D \\
& -C_{1}\left(1-y^{2}+\frac{2}{M}+2 \mathrm{Kn}\right),
\end{aligned}
$$

where

$$
\begin{aligned}
D & =\Phi_{f 20}^{\prime}( \pm 1)=-\frac{1}{2}\left[( A _ { 1 } + A _ { 1 } ^ { * } ) \left(\alpha^{2} \sinh (\alpha)\right.\right. \\
& \left.+\alpha^{3} \mathrm{Kn} \cosh (\alpha)\right)+B_{1}\left(\beta^{2} \sinh (\beta)\right.
\end{aligned}
$$

$$
\begin{aligned}
+ & \left.\beta^{3} \operatorname{Kn} \cosh (\beta)\right)+B_{1}^{*}\left(\beta^{* 2} \sinh \left(\beta^{*}\right)\right. \\
+ & \left.\left.\beta^{* 3} \cosh \left(\beta^{*}\right)\right)\right] \\
F(y) & =\frac{(1-C)^{2} \alpha^{2} R^{2}}{4}\left[\gamma^{2}\left(A_{1}^{*} B_{1}+\frac{C M A_{2}^{*} B_{2}}{(1-C) N}\right)\right. \\
\times & {\left[\frac{\cosh (\alpha+\beta) y}{(\alpha+\beta)^{2}}-\frac{\cosh (\alpha-\beta) y}{(\alpha-\beta)^{2}}\right] } \\
+ & \gamma^{* 2}\left(A_{1} B_{1}^{*}+\frac{C M A_{2} B_{2}^{*}}{(1-C) N}\right) \times\left[\frac{\cosh \left(\alpha+\beta^{*}\right) y}{\left(\alpha+\beta^{*}\right)^{2}}\right. \\
- & \left.\frac{\cosh \left(\alpha-\beta^{*}\right) y}{\left(\alpha-\beta^{*}\right)^{2}}\right]+\left(\gamma^{2}+\gamma^{* 2}\right) \times\left(B_{1} B_{1}^{*}\right.
\end{aligned}
$$




$$
\begin{aligned}
& \left.+\frac{C M B_{2} B_{2}^{*}}{(1-C) N}\right)\left[\frac{\cosh \left(\beta+\beta^{*}\right) y}{\left(\beta+\beta^{*}\right)^{2}}\right. \\
& \left.\left.-\frac{\cosh \left(\beta-\beta^{*}\right) y}{\left(\beta-\beta^{*}\right)^{2}}\right]\right], \\
& G(y)=F(y) \\
& -\frac{(1-C)^{2} \alpha^{2} R^{2}}{4 N}\left[\gamma^{2} A_{2}^{*} B_{2}[\cosh (\alpha+\beta) y\right. \\
& -\cosh (\alpha-\beta) y]+\gamma^{* 2} A_{2} B_{2}^{*}\left[\cosh \left(\alpha+\beta^{*}\right) y\right. \\
& \left.-\cosh \left(\alpha-\beta^{*}\right) y\right]+\left(\gamma^{2}+\gamma^{* 2}\right) \\
& \left.\cdot B_{2} B_{2}^{*}\left[\cosh \left(\beta+\beta^{*}\right)-\cosh \left(\beta-\beta^{*}\right)\right]\right],
\end{aligned}
$$

with

$$
\gamma^{2}=1+\frac{C M}{(1-C)(N-i \alpha R)} .
$$

Thus, we see that one constant $C_{1}$ remains arbitrary in the solution which is found to be proportional to the secondorder time-averaged pressure gradient. If we time-average (1) for the solution given by (1), (2), (4), (5), (23), (24), (25), ((29a), (29b)), ((30a), (30b)), (39), and (41), we find that

$$
c_{1}=R\left(\frac{\overline{\partial p}}{\partial x}\right)_{2} .
$$

The constant $c_{1}$, which is related to the second-order pressure gradient distribution, may be obtained using ends conditions of the real physical problem.

The mean time average velocities may now be written as

$$
\begin{aligned}
\bar{u}_{f} & =\frac{\varepsilon^{2}}{2} \Phi_{f 20}^{\prime}(y)=\frac{\varepsilon^{2}}{2}[F(y)-F(1)+D \\
- & \left.R\left(\frac{\overline{\partial p}}{\partial x}\right)_{2}\left(1-y^{2}+2 \mathrm{Kn}\right)\right], \\
\bar{u}_{p} & =\frac{\varepsilon^{2}}{2} \Phi_{p 20}^{\prime}(y)=\frac{\varepsilon^{2}}{2}[G(y)-F(1)+D \\
& \left.-R\left(\frac{\overline{\partial p}}{\partial x}\right)_{2}\left(1-y^{2}+\frac{2}{M}+2 \mathrm{Kn}\right)\right] .
\end{aligned}
$$

If no-slip, that is, $\mathrm{Kn}=0$, results of the present problems reduce exactly to the same as that found by L. M. Srivastava and V. P. Srivastava [5].

Also if no-slip, that is, $\mathrm{Kn}=0$ and the fluid is particles free, that is, $C=0$, results of the present problems reduce exactly to the same as that found by Fung and Yih [34].

\section{Numerical Results and Discussion}

A close look at (45) reveals that the mean axial velocity of the fluid phase $\bar{u}_{f}$ is dominated by the constant $D$ and the parabolic distribution term $-R(\partial p / \partial x)_{2}\left(l-y^{2}+2 \mathrm{Kn}\right)$. The term $F(y)-F(1)$, always a negative quantity, is negligible compared to $D$. The constant $D$, which initially arose from the slip condition of the axial velocity on the wall, is due to the value of $\Phi_{f 20}$ at the boundary and is related to the mean velocity at the boundaries of the channel (at $y= \pm 1$ ) by $\bar{u}=\left(\varepsilon^{2} / 2\right)\left(D-R(\partial p / \partial x)_{2}(2 \mathrm{Kn})\right)$. This shows that the slip boundary condition applies to the wavy wall and not to the mean position of the wall. It may be reminded here that the corresponding $D$ does not appear in the particulate phase mean axial velocity as the particulate phase velocity at the walls was unspecified.

For the sake of comparison we define mean-velocity perturbation function $f(y)$ in accordance with Fung and Yih [34] and L. M. Srivastava and V. P. Srivastava [5] as

$$
f=-\frac{200}{\alpha^{2} R^{2}}(F(y)-F(1)),
$$

which gives mean time axial velocity of the fluid phase as

$$
\begin{aligned}
& \bar{u}_{f}(y)=\frac{\varepsilon^{2}}{2}\left(D-R\left(\frac{\overline{\partial p}}{\partial x}\right)_{2}\left(1-y^{2}+2 \mathrm{Kn}\right)\right. \\
& \left.-\frac{\alpha^{2} R^{2}}{200} f(y)\right) .
\end{aligned}
$$

It has been observed that urine, bacteria, or other materials some time pass from the bladder to the kidney or from one kidney to the other in direction opposite to the urine flow. Physiologists term these phenomena as "ureteral reflux." Two different definitions of reflux exist in the literature; Shapiro et al. [40] call a flow reflux whenever there is a negative net displacement of a particle trajectory, while Yin and Fung [41] define a flow reflux whenever there is a negative mean velocity in the flow field. In the present analysis the latter definition of reflux is adopted as L. M. Srivastava and V. P. Srivastava [5].

Since $D$ is always a positive quantity, $\bar{u}=\left(\varepsilon^{2} / 2\right) D$ at $y=$ \pm 1 shows that the mean flow reversal will never occur at the boundaries. Further, from (48), it is clear that the reflux would occur when the mean pressure gradient $(\partial p / \partial x)_{2}$ reaches a certain critical value. Thus, the critical reflux condition may be defined as one for which the mean velocity $\bar{u}(y)$ is equal to zero on the center line $y=0$; (48) yields

$$
\begin{aligned}
\frac{C_{1}}{R} & =\left(\frac{\overline{\partial p}}{\partial x}\right)_{2 \text { critical }} \\
& =\frac{1}{R(1+2 \mathrm{Kn})}\left(D-\frac{\alpha^{2} R^{2}}{200} f(0)\right) .
\end{aligned}
$$

For $(\overline{\partial p} / \partial x)_{2}<(\overline{\partial p} / \partial x)_{2 \text { critical reflux }}$, there is no reflux and if $(\overline{\partial p} / \partial x)_{2}>(\overline{\partial p} / \partial x)_{2}$ critical reflux there will be reflux and a backward flow in the neighborhood of the center line occurs.

The value of $(\overline{\partial p} / \partial x)_{2}$ critical reflux for various values of $C$, $R, \mathrm{Kn}$, and $\alpha$ is displayed in Figure 2. From the figure, the results reveal that the value of $(\overline{\partial p} / \partial x)_{2 \text { critical reflux }}$ with $y$ for different values of $C$, for a fixed value of $R,(\overline{\partial p} / \partial x)_{2 \text { critical reflux }}$ 


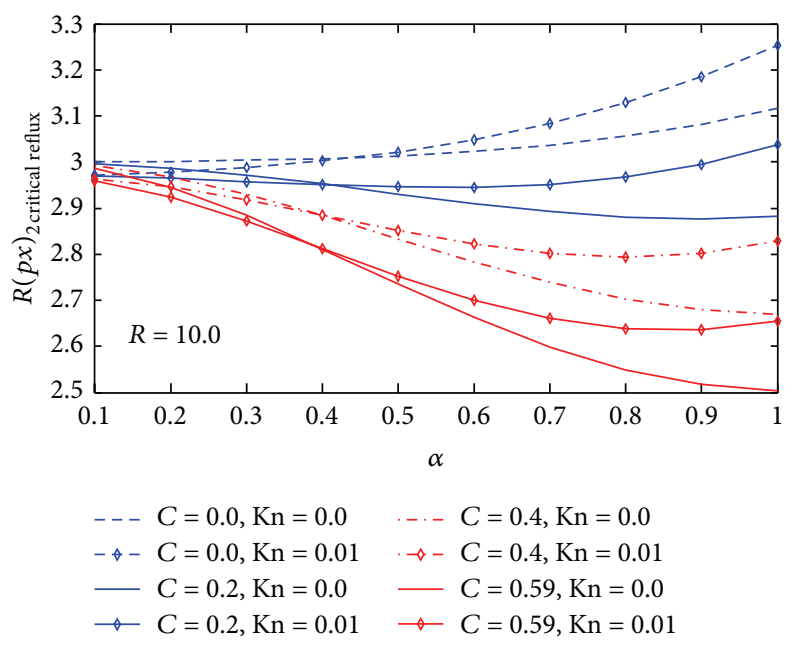

FIGURE 2: Effect of the Knudsen number Kn, particle concentration $C$, and wave number $\alpha$ on the critical reflux pressure gradient at $R=10$.

decreases with increasing particle concentration $C$. However, $C$ have significant influence over $(\overline{\partial p} / \partial x)_{2}$ critical reflux only at higher values of $\alpha$. In the presence of Knudsen number Kn, we observed that, at $\alpha \leq 0.4,(\overline{\partial p} / \partial x)_{2 \text { critical reflux }}$ decreases with increasing wave number $\alpha$. However, $\alpha>0.4$ and $(\overline{\partial p} / \partial x)_{2}$ critical reflux increases with increasing wave number $\alpha$. We observed that the critical reflux pressure $(\overline{\partial p} / \partial x)_{2 \text { critical reflux }}$ at a given $\alpha$ and $R$ is lower for particlefluid suspension than for particle-free fluid. This means that presence of particle in the fluid favors reversal flow.

Finally, in Figures 3-6, the mean-velocity distribution with reversal flow is displayed. Effects of $C, R, \mathrm{Kn}$, and $(\overline{\partial p} / \partial x)_{2}$ on mean velocity and reversal flow are shown.

Figure 7 studies the effect of Knudsen number Kn and the mean second-order pressure gradient $(\partial p / \partial x)_{2}$ on the meanvelocity distribution and reversal flow for $C=0.4, \alpha=1.0$, and $R=10$; we notice that the mean-velocity distribution increases with increasing Knudsen number Kn forward for $(\overline{\partial p} / \partial x)_{2}<(\overline{\partial p} / \partial x)_{2 \text { critical reflux }}$ while for $(\overline{\partial p} / \partial x)_{2}>$ $(\overline{\partial p} / \partial x)_{2}$ critical reflux the velocity increases the reflux flow. Also we notice that the mean-velocity distribution decrease with increasing the value of $(\overline{\partial p} / \partial x)_{2}$.

Also we notice that the value of $(\overline{\partial p} / \partial x)_{2}$ critical reflux decreases by increasing the Knudsen number Kn.

Figure 4 studies the effect of Knudsen number Kn and particle concentration $C$ on the mean-velocity distribution mean and reversal flow for $\alpha=1.0,(\overline{\partial p} / \partial x)_{2}=0.3$, and $R=10$, and the figures reveal that the reversal flow increases with increasing particle concentration $C$, but the presence of Knudsen number Kn results in a decrease in the reversal flow. Also we notice that the mean-velocity distribution increases with increasing $\mathrm{Kn}$. Interpreted physiologically, this means that, under the same conditions, urine in which solute particles are suspended (i.e., urine from a diseased kidney) is more susceptible to reversal flow in ureter, in comparison to pure urine without solute particles.
Figure 5 studies the effect of Knudsen number Kn and the Reynolds number $R$ on the mean-velocity distribution and reversal flow for $\alpha=0.05,(\overline{\partial p} / \partial x)_{2}=0.5$, and $C=$ 0.3 , and the figures reveal that the reversal flow increases with increasing Reynolds number $R$. Also we notice that from Figures 5(a) and 5(b), at $R<3$, the presence of Knudsen number Kn results a decrease in the mean-velocity distribution, from Figure 5(c) at $R=3$, the effect of Knudsen number vanishes, with increasing $\mathrm{Kn}$ at $R>3$, and we observed that the presence of Knudsen number Kn results in increase in the mean-velocity distribution and the reversal flow. Figure 6 studies the effect of Knudsen number Kn and the wave number $\alpha$ on the mean-velocity distribution and reversal flow for $R=10,(\overline{\partial p} / \partial x)_{2}=0.5$, and $C=0.3$; the figures reveal that the reversal flow increases with increasing wave number $\alpha$. Also we notice that from Figures 6(a) and $6(\mathrm{~b})$, at $\alpha<0.6$, the presence of Knudsen number Kn results in an increase in the reversal flow, from Figure 6(c) at $\alpha=0.6$, the effect of Knudsen number vanishes, with increasing $\alpha$ at $\alpha>0.6$, and we observed that the presence of Knudsen number Kn results in decrease in the reversal flow.

Next, we return to the dimensional flow problem; the dimensional mean axial velocity $\left\langle u_{f}\right\rangle$ is equal to the dimensionless mean net axial velocity $\bar{u}_{f}$ as given by (48) multiplied by the factor $c$. The properties of the blood are given by $\rho_{f}=1066 \mathrm{~kg} / \mathrm{m} 3$ and $\mu=4 \times 10^{-3} \mathrm{Nm}^{-2} \mathrm{~s}$. The particle concentration $C$ various accurate up to $C=0.6$ [39]. The frequency $f$ of the wave is related to the wave speed $c$ and the wavelength $\lambda$ according to $\lambda=c / f$.

According to the Knudsen number, the flow regimes can be divided into various regions. These are continuum, slip, transition, and free molecular flow regimes. If $\mathrm{Kn}<0.001$, so that molecular mean free path of the molecules is negligible in comparison to the geometrical dimensions, the fluid can be treated as a continuous medium. If $0.001<\mathrm{Kn}<0.1$, it is found that the fluid loses grip on the boundaries and tends to slip along the walls of the domain. If $0.1<\mathrm{Kn}<3.0$, 

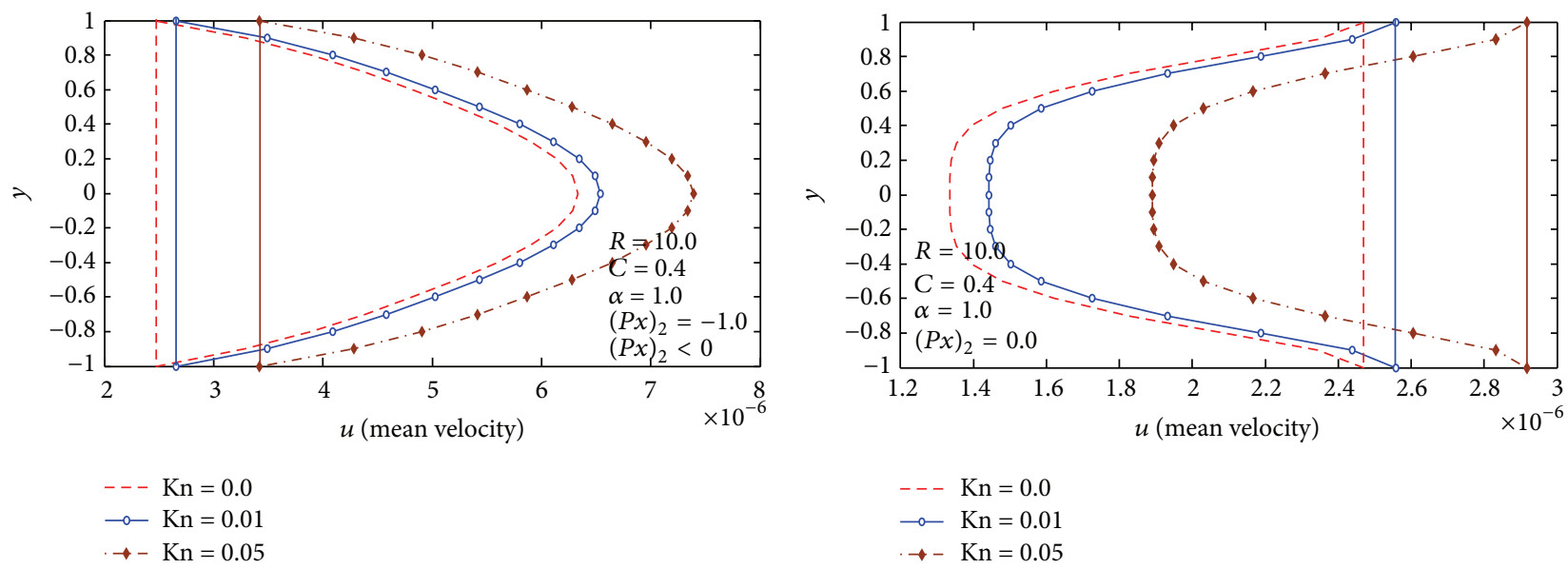

(a)

(b)
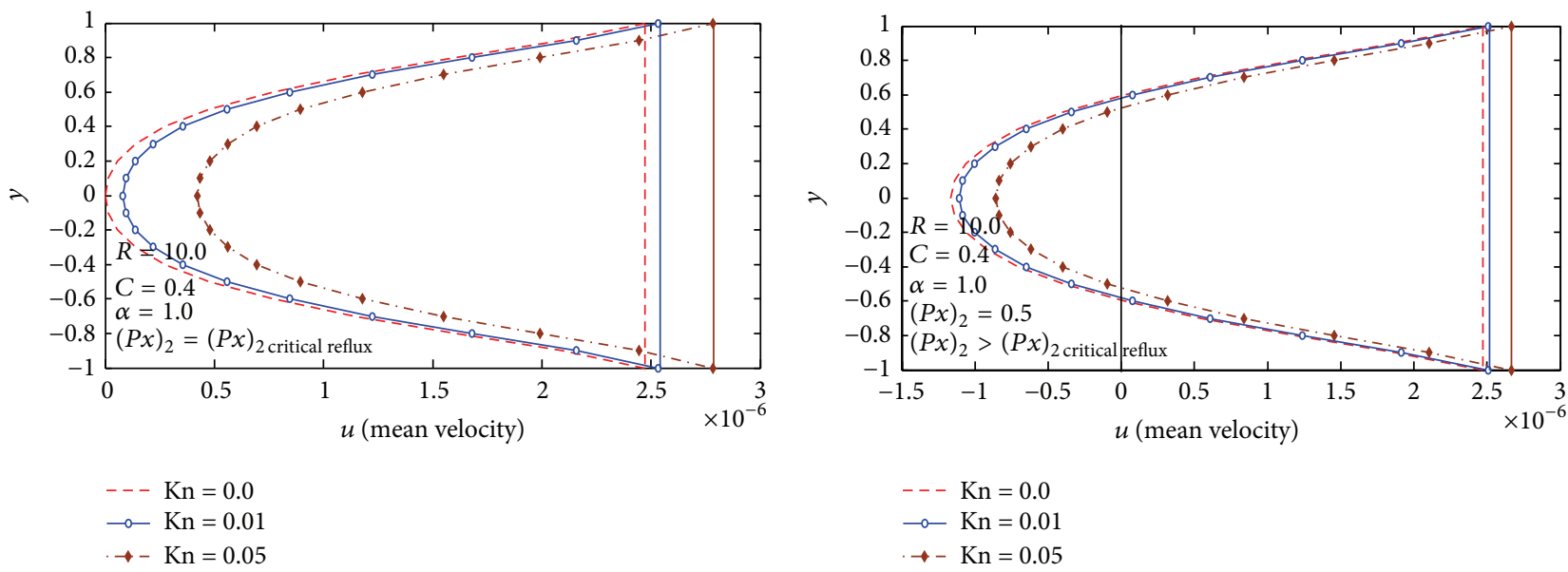

(c)

(d)

Figure 3: (a) Mean velocity distribution at $(\partial p / \partial x)_{2}=-1.0$. (b) Mean velocity distribution at $(\partial p / \partial x)_{2}=0.0$. (c) Mean velocity distribution at $(\partial p / \partial x)_{2}=(\partial p / \partial x)_{2 \text { Critical reflux. }}$ (d) Mean velocity distribution at $(\partial p / \partial x)_{2}=0.5$.

it is transition flow regimes. Finally, the flow enters the free molecular regime when $\mathrm{Kn}>3.0$, each requiring a particular type of analysis [21, 23].

For example, for the left main coronary artery, the range of the diameter is $2.0-5.5 \mathrm{~mm}$ (mean $4 \mathrm{~mm}$ ) and wavelength range $\lambda=2.512-12.56 \mathrm{~cm}$ [39]. The dimensional mean axial velocity $\left\langle u_{f}\right\rangle(\mathrm{m} / \mathrm{s})$ is plotted versus $y(m)$, for $\lambda=$ $12.56 \mathrm{~cm}$, the half of mean width $d=2.0 \mathrm{~mm}$, the wave has amplitude $a_{0}=10^{-4} \mathrm{~mm}$, and $C=0.3$, with various values of Knudsen number $\mathrm{Kn}$, for $\mathrm{Kn}=0.0, \mathrm{Kn}=0.01, \mathrm{Kn}=0.03$, $\mathrm{Kn}=0.06$, and $\mathrm{Kn}=0.1$. We observe that, from Figure 7(a), for $(\overline{\partial p} / \partial x)_{2}<(\overline{\partial p} / \partial x)_{2}$ critical reflux $\left((\overline{\partial p} / \partial x)_{2}=-1.0\right)$, the mean axial velocity $\left\langle u_{f}\right\rangle$ increases with increasing Knudsen number Kn and there is no reflux flow forward for $(\overline{\partial p} / \partial x)_{2}<$ $(\overline{\partial p} / \partial x)_{2}$ critical reflux while, from Figure $7(\mathrm{~b})$, for $(\overline{\partial p} / \partial x)_{2}>$ $(\overline{\partial p} / \partial x)_{2 \text { critical reflux }}\left((\overline{\partial p} / \partial x)_{2}=0.5\right)$ there will be reflux flow and a backward flow in the neighborhood of the center line occurring, and the mean axial velocity $\left\langle u_{f}\right\rangle$ increases in the reversal flow with increasing Knudsen number Kn.

\section{Conclusions}

There is not any attempt to study the effect of slip conditions on the flow of a particle-fluid suspension with peristalsis. The purpose of this paper is to study the slip effects on the peristaltic pumping of a particle-fluid mixture in a two-dimensional channel. It is an application of the twodimensional analysis of peristaltic motion of a particle-fluid mixture by L. M. Srivastava and V. P. Srivastava [5] and the two-dimensional analysis of peristaltic motion of single phase fluid by Fung and Yih [34] in the presence of slip effects. The mathematical model considers a particle-fluid mixture between infinite parallel walls with slip condition on which a sinusoidal traveling wave is imposed. A perturbation solution is obtained which satisfies the momentum equations for the case in which amplitude ratio (wave amplitude/channel half width) is small. Finally, the phenomenon of the mean flow reversal is presented and its physiological implication is discussed. Beside the engineering applications of pumping particle-fluid mixture by peristalsis, the present analysis of 

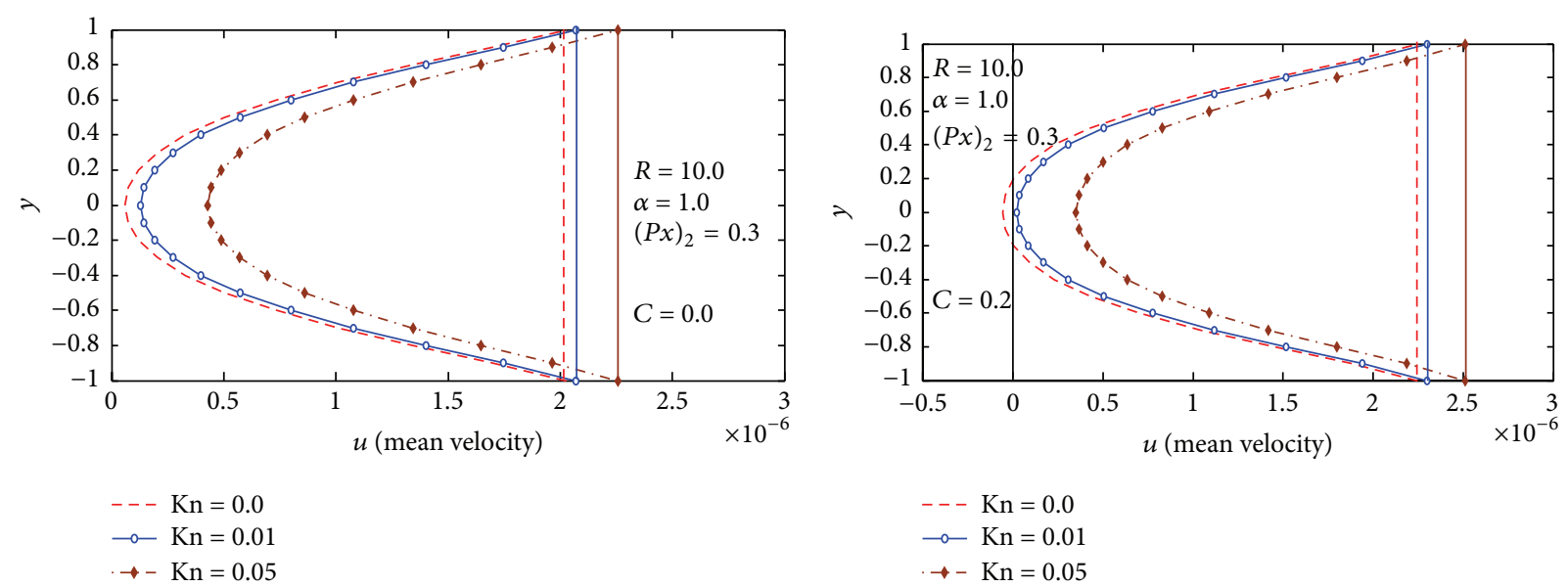

(a)

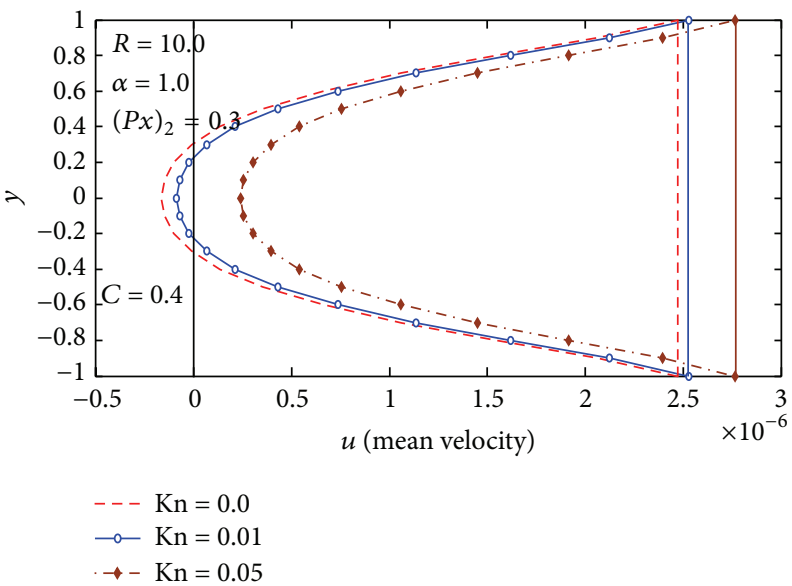

(c)

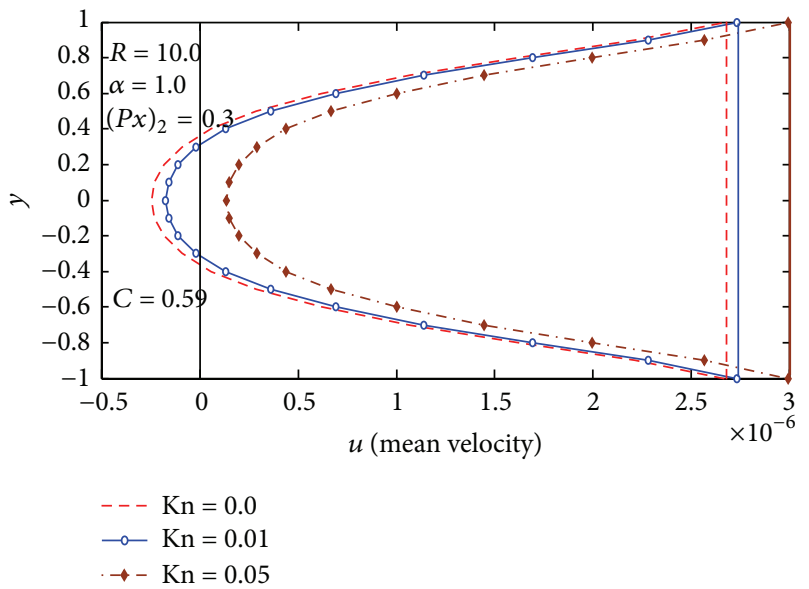

(d)

Figure 4: (a) Mean velocity distribution at $C=0.0, \alpha=1.0,(\overline{\partial p} / \partial x)_{2}=0.3$, and $R=10$. (b) Mean velocity distribution at $C=0.2, \alpha=1.0$, $(\overline{\partial p} / \partial x)_{2}=0.3$, and $R=10$. (c) Mean velocity distribution at $C=0.4, \alpha=1.0,(\overline{\partial p} / \partial x)_{2}=0.3$, and $R=10$. (d) Mean velocity distribution at $C=0.59, \alpha=1.0,(\overline{\partial p} / \partial x)_{2}=0.3$, and $R=10$.

two-phase flow process is potentially important in regard to biofluid transport by peristalsis muscular contractions in body organs where fluids behave like particle-fluid mixtures, namely, chime in small intestine, spermatic fluid in cervical canal, urine (from a diseased kidney) in ureter, and blood suspension in arteriole.

Some concluding remarks are as follows.

(i) The reversal flow increases with increasing particle concentration $C$, but the presence of Knudsen number $\mathrm{Kn}$ results in a decrease in the reversal flow. The presence of Knudsen number Kn results in a decrease in the reversal flow.

(ii) Also we notice that the mean-velocity distribution increases with increasing Kn. Interpreted physiologically, this means that, under some conditions, urine in which solute particles are suspended (i.e., urine from a diseased kidney) is more susceptible to reversal flow in ureter, in comparison to pure urine without solute particles. (iii) For example, for the left main coronary artery, the mean axial velocity $\left\langle u_{f}\right\rangle$ increases with increasing Knudsen number $\mathrm{Kn}$ and there is no reflux flow forward for $(\overline{\partial p} / \partial x)_{2}<(\overline{\partial p} / \partial x)_{2}$ critical reflux while for $(\overline{\partial p} / \partial x)_{2}>(\overline{\partial p} / \partial x)_{2 \text { critical reflux }}$ there will be reflux and a backward flow in the neighborhood of the center line occurring, and the mean axial velocity $\left\langle u_{f}\right\rangle$ increases in the reversal flow with increasing Knudsen number Kn.

Comparing with other models for verifications of results, the present model gives the most general form of velocity expression from which the other mathematical models can easily be obtained by proper substitutions. It is of interest to note that the result of the present model includes results of different mathematical models such as the following.

(1) The results of L. M. Srivastava and V. P. Srivastava [5] have been recovered by taking Knudsen number $\mathrm{kn}=$ 0.0 (no-slip condition). 


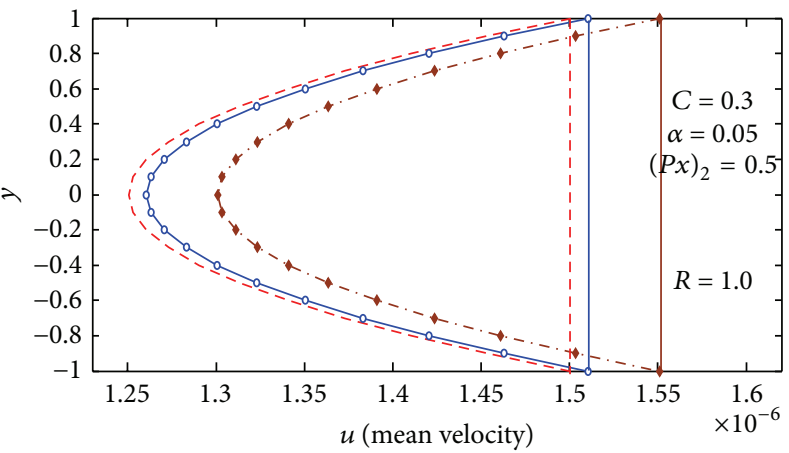

(a)

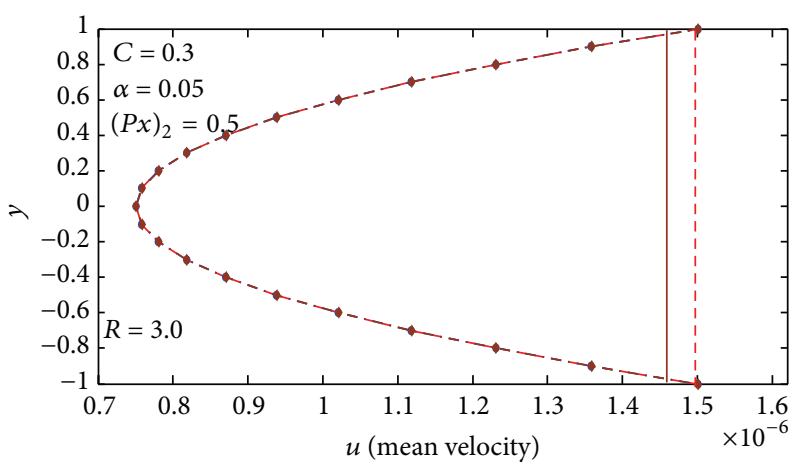

(c)

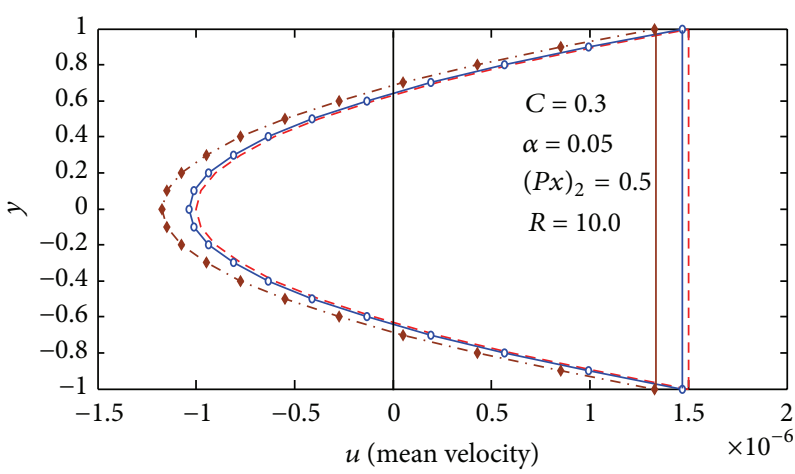

(e)

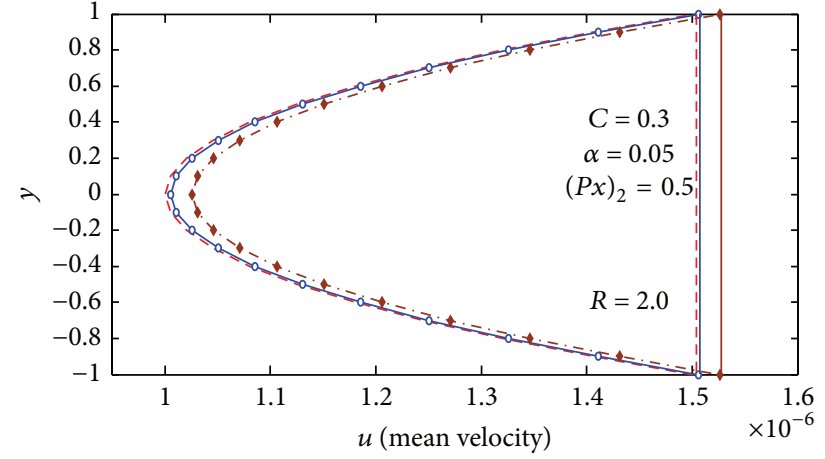

(b)

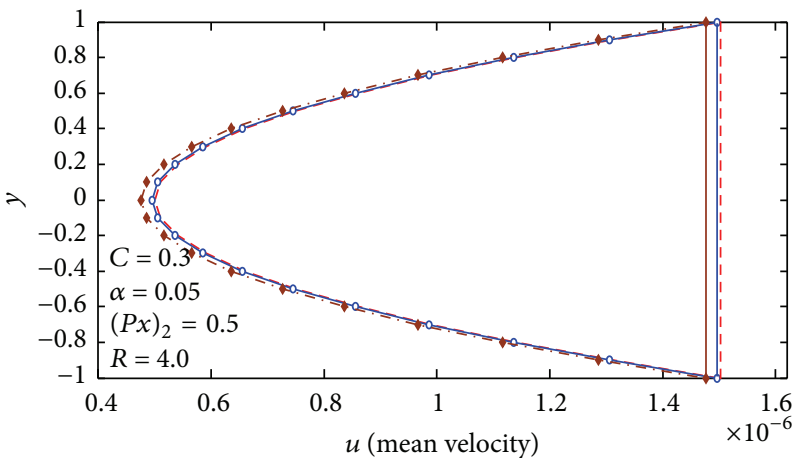

(d)

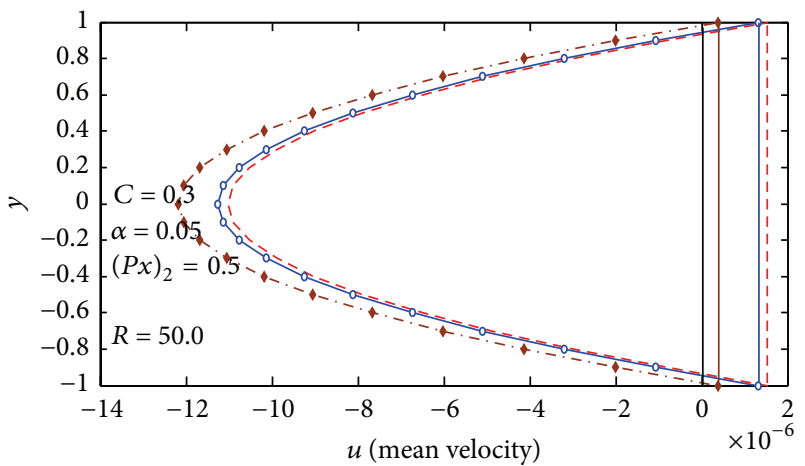

(f)

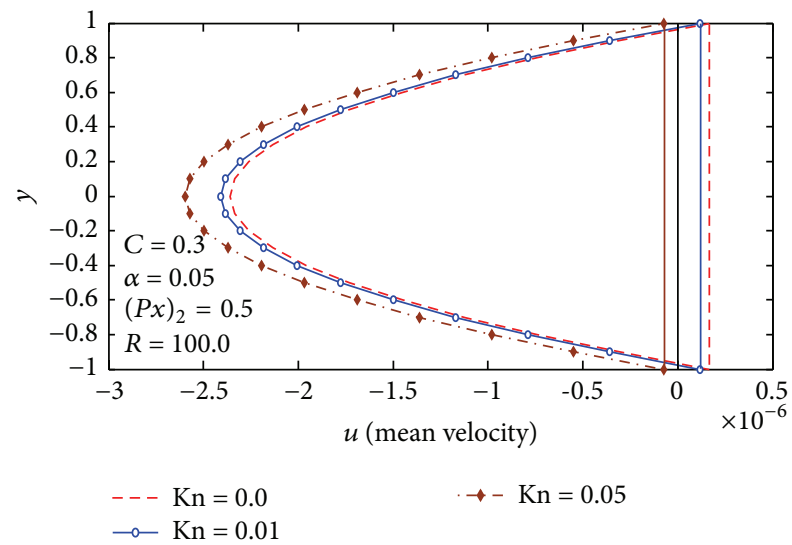

(g)

Figure 5: (a) Mean velocity distribution at $R=1.0, \alpha=0.05,(\overline{\partial p} / \partial x)_{2}=0.5$, and $C=0.3$. (b) Mean velocity distribution at $R=2.0, \alpha=0.05$, $(\overline{\partial p} / \partial x)_{2}=0.5$, and $C=0.3$. (c) Mean velocity distribution at $R=3.0, \alpha=0.05,(\overline{\partial p} / \partial x)_{2}=0.5$, and $C=0.3$. (d) Mean velocity distribution at $R=4.0, \alpha=0.05,(\overline{\partial p} / \partial x)_{2}=0.5$, and $C=0.3$. (e) Mean velocity distribution at $R=10.0, \alpha=0.05,(\overline{\partial p} / \partial x)_{2}=0.5$, and $C=0.3$. (f) Mean velocity distribution at $R=50.0, \alpha=0.05,(\overline{\partial p} / \partial x)_{2}=0.5$, and $C=0.3$. (g) Mean velocity distribution at $R=100.0, \alpha=0.05,(\overline{\partial p} / \partial x)_{2}=0.5$, and $C=0.3$. 


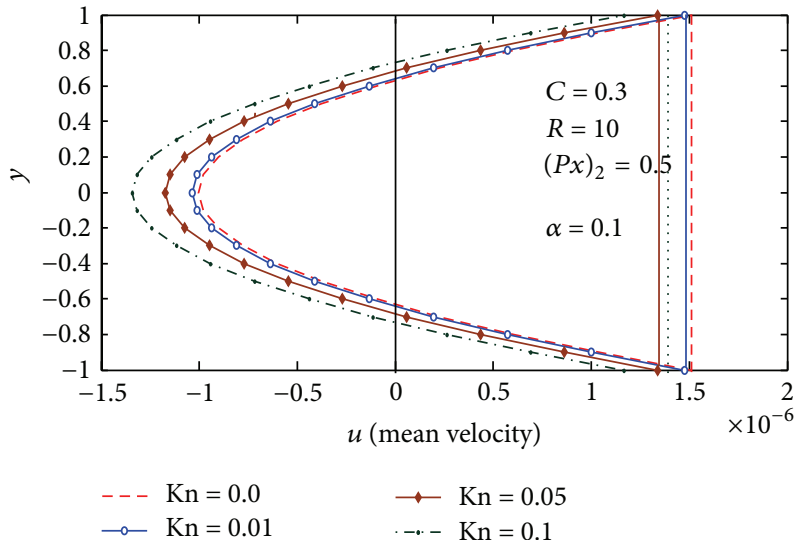

(a)

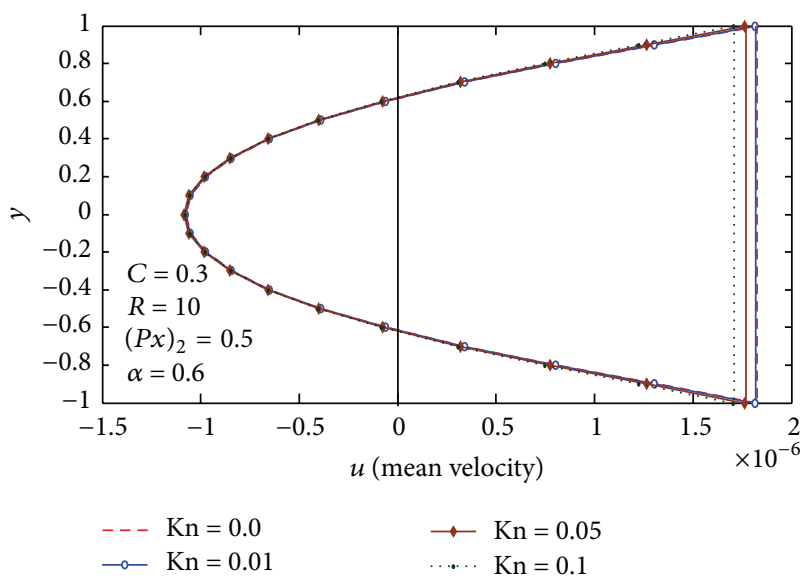

(c)

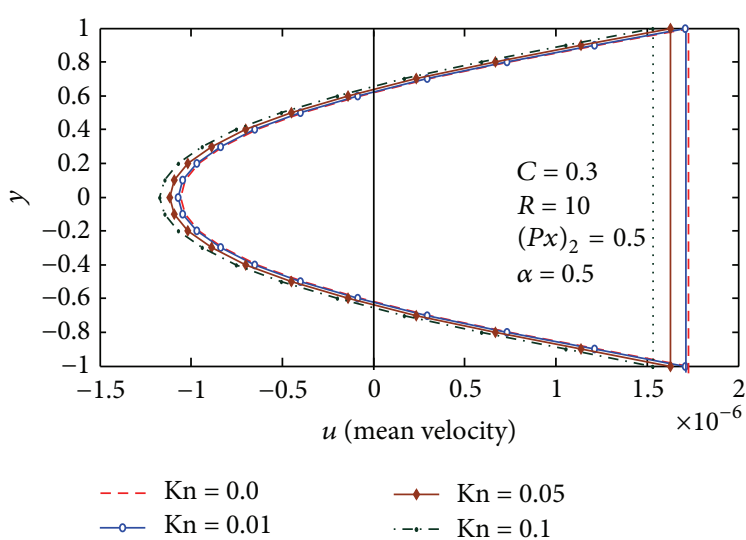

(b)

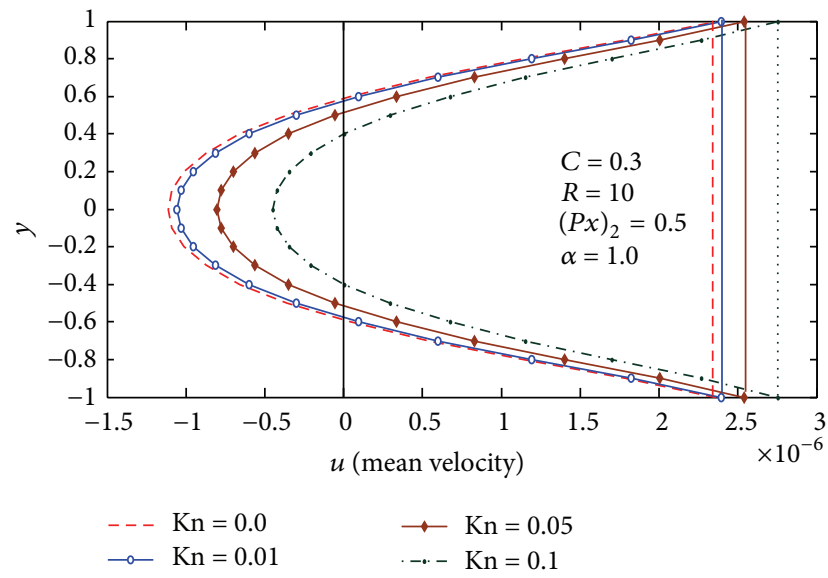

(d)

Figure 6: (a) Mean velocity distribution at $\alpha=0.1,(\overline{\partial p} / \partial x)_{2}=0.5$, and $C=0.3$. (b) Mean velocity distribution at $\alpha=0.5,(\overline{\partial p} / \partial x)_{2}=0.5$, and $C=0.3$. (c) Mean velocity distribution at $\alpha=0.6,(\overline{\partial p} / \partial x)_{2}=0.5$, and $C=0.3$. (d) Mean velocity distribution at $\alpha=1.0,(\overline{\partial p} / \partial x)_{2}=0.5$, and $C=0.3$.

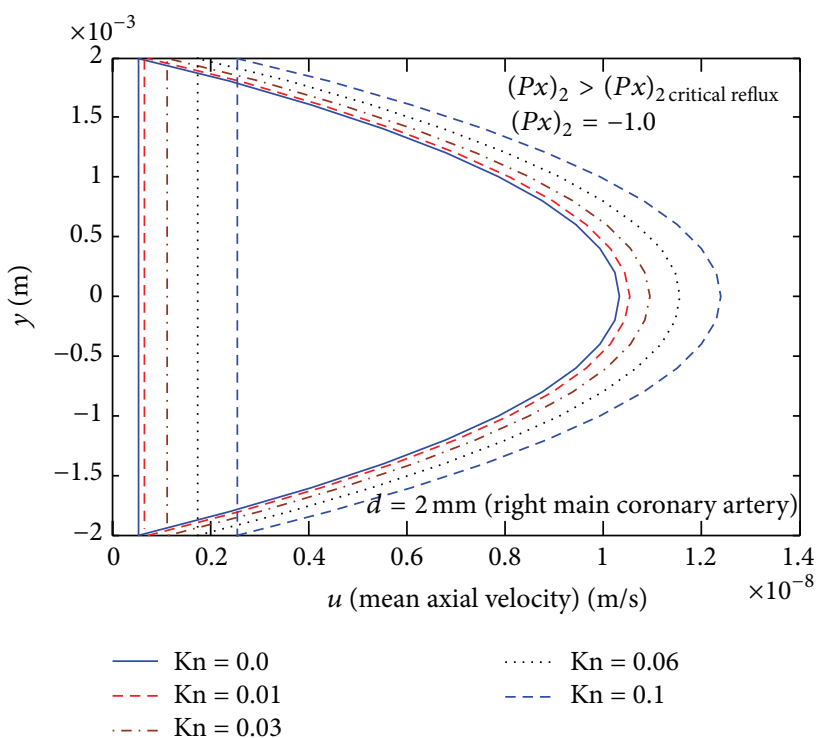

(a)

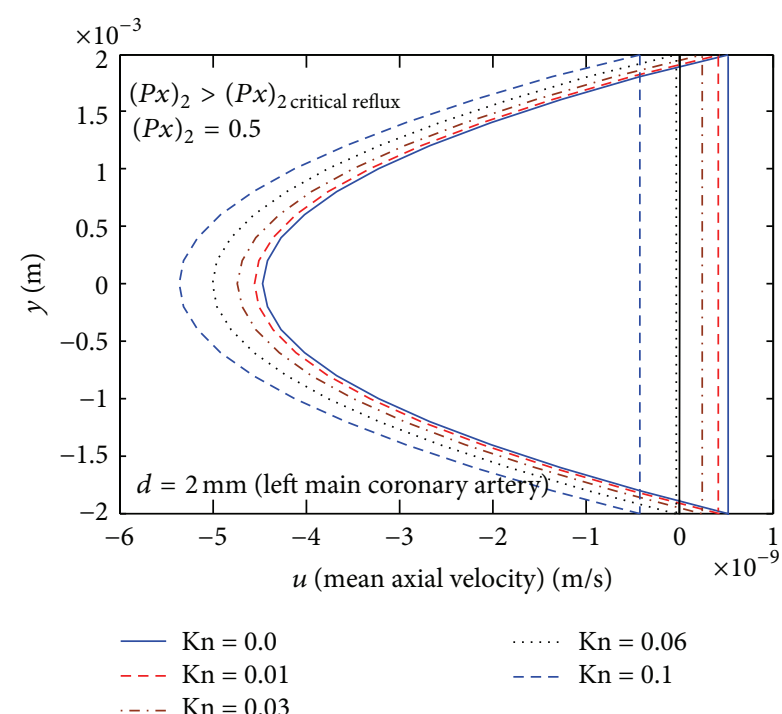

(b)

Figure 7: (a) Mean axial velocity $(\mathrm{m} / \mathrm{s})$ distribution at $\left.(\overline{\partial p} / \partial x)_{2}<(\overline{\partial p} / \partial x)_{2 \text { critical reflux }}(\overline{\partial p} / \partial x)_{2}=-1.0\right)$. (b) Mean axial velocity $(\mathrm{m} / \mathrm{s})$ distribution at $(\overline{\partial p} / \partial x)_{2}>(\overline{\partial p} / \partial x)_{2 \text { critical reflux }}\left((\overline{\partial p} / \partial x)_{2}=0.5\right)$. 
(2) The results of Fung and Yih [34] have been recovered by taking Knudsen number $\mathrm{kn}=0.0$ and the fluid is particles-free; that is, $C=0$.

\section{Conflict of Interests}

The authors declare that there is no conflict of interests regarding the publication of this paper.

\section{References}

[1] T. W. Latham, Fluid motion in a peristaltic pump [M.S. thesis], Massachusetts Institute of Technology, Cambridge, Mass, USA, 1966.

[2] H. J. Rath, Peristaltische Strömungen, Springer, Berlin, Germany, 1980.

[3] L. M. Srivastava and V. P. Srivastava, "Peristaltic transport of blood: casson model-II," Journal of Biomechanics, vol. 17, no. 11, pp. 821-829, 1984.

[4] L. M. Srivastava and V. P. Srivastava, "Interaction of peristaltic flow with pulsatile flow in a circular cylindrical tube," Journal of Biomechanical Engineering, vol. 18, no. 4, pp. 247-253, 1985.

[5] L. M. Srivastava and V. P. Srivastava, "Peristaltic transport of a particle-fluid suspension," Journal of Biomechanical Engineering, vol. 111, no. 2, pp. 157-165, 1989.

[6] V. P. Srivastava and M. Saxena, "A two-fluid model of nonNewtonian blood flow induced by peristaltic waves," Rheologica Acta, vol. 34, no. 4, pp. 406-414, 1995.

[7] V. P. Srivastava and L. M. Srivastava, "Effects of Poiseuille flow on peristaltic transport of a particulate suspension," Zeitschrift für Angewandte Mathematik und Physik, vol. 46, no. 5, pp. 655679, 1995.

[8] C. D. Hill and A. Bedford, "A model for erythrocyte sedimentation," Biorheology, vol. 18, no. 2, pp. 255-266, 1981.

[9] L. M. Srivastava and V. P. Srivastava, "On two-phase model of pulsatile blood flow with entrance effects," Biorheology, vol. 20, no. 6, pp. 761-777, 1983.

[10] E. A. Trowbridge, "The fluid mechanics of blood," in Mathematics in Medicine and Biomechanics, vol. 7 of Shiva Mathematics Series, pp. 200-217, University of Strathclyde Seminars in Applied Mathematical Analysis, 1984.

[11] S. Oka, "A physical theory of erythrocyte sedimentation," Biorheology, vol. 22, no. 4, pp. 315-321, 1985.

[12] H. Wang and R. Skalak, "Viscous flow in a cylindrical tube containing a line of spherical particles," Journal of Fluid Mechanics, vol. 38, pp. 75-96, 1970.

[13] P. Bungay and H. Brenner, "Pressure drop due to the motion of a sphere near the wall bounding a Poiseuille flow," Journal of Fluid Mechanics, vol. 60, pp. 81-96, 1973.

[14] R. Skalak, P. H. Chen, and S. Chien, "Effect of hematocrit and rouleaux on apparent viscosity in capillaries," Biorheology, vol. 9, no. 2, pp. 67-82, 1972.

[15] T. Karino, H. H. M. Kwong, and H. L. Goldsmith, "Particle flow behaviour in models of branching vessels: I. Vortices in $90^{\circ} \mathrm{T}$ junctions," Biorheology, vol. 16, no. 3, pp. 231-248, 1979.

[16] T.-K. Hung and T. D. Brown, "Solid-particle motion in twodimensional peristaltic flows," Journal of Fluid Mechanics, vol. 73, no. 1, pp. 77-96, 1976.

[17] T. D. Brown and T.-K. Hung, "Computational and experimental investigations of two-dimensional nonlinear peristaltic flows," Journal of Fluid Mechanics, vol. 83, no. 2, pp. 249-272, 1977.
[18] S. Takabatake and K. Ayukawa, "Numerical study of twodimensional peristaltic flows," Journal of Fluid Mechanics, vol. 122, pp. 439-465, 1982.

[19] K. S. Mekheimer, E. F. El Shehawey, and A. M. Elaw, "Peristaltic motion of a particle-fluid suspension in a planar channel," International Journal of Theoretical Physics, vol. 37, no. 11, pp. 2895-2920, 1998.

[20] A. M. El Misery, E. F. Elshehawey, and A. A. Hakeem, "Peristaltic motion of an incompressible generalized Newtonian fluid in a planar channel," Journal of the Physical Society of Japan, vol. 65, no. 11, pp. 3524-3529, 1996 (Japanese).

[21] E. F. El-Shehawy, N. T. El-Dabe, and I. M. El-Desoky, "Slip effects on the peristaltic flow of a non-Newtonian Maxwellian fluid," Journal of Acta Mechanica, vol. 186, no. 1-4, pp. 141-159, 2006.

[22] I. M. Eldesoky, "Influence of slip condition on peristaltic transport of a compressible Maxwell fluid through porous medium in a tube," International Journal of Applied Mathematics and Mechanics, vol. 8, no. 2, pp. 99-117, 2012.

[23] W. Kwang-Hua Chu and J. Fang, "Peristaltic transport in a slip flow," The European Physical Journal B, vol. 16, no. 3, pp. 543$547,2000$.

[24] N. Ali, Q. Hussain, T. Hayat, and S. Asghar, "Slip effects on the peristaltic transport of MHD fluid with variable viscosity," Physics Letters A, vol. 372, no. 9, pp. 1477-1489, 2008.

[25] T. Hayat, M. U. Qureshi, and N. Ali, "The influence of slip on the peristaltic motion of a third order fluid in an asymmetric channel," Physics Letters A, vol. 372, no. 15, pp. 2653-2664, 2008.

[26] A. M. Sobh, "Interaction of couple stresses and slip flow on peristaltic transport in uniform and nonuniform channels," Turkish Journal of Engineering and Environmental Sciences, vol. 32, no. 2, pp. 117-123, 2008.

[27] A. M. Sobh, "Slip flow in peristaltic transport of a Carreau fluid in an asymmetric channel," Canadian Journal of Physics, vol. 87, no. 8, pp. 957-965, 2009.

[28] S. Nadeem and S. Akram, "Slip effects on the peristaltic flow of a Jeffrey fluid in an asymmetric channel under the effect of induced magnetic field," International Journal for Numerical Methods in Fluids, vol. 63, no. 3, pp. 374-394, 2010.

[29] M. El-Shahed, "Pulsatile flow of blood through a stenosed porous medium under periodic body acceleration," Applied Mathematics and Computation, vol. 138, no. 2-3, pp. 479-488, 2003.

[30] I. M. Eldesoky, "Slip effects on the unsteady MHD pulsatile blood flow through porous medium in an artery under the effect of body acceleration," International Journal of Mathematics and Mathematical Sciences, vol. 2012, Article ID 860239, 26 pages, 2012.

[31] I. M. Eldesoky, M. H. Kamel, and R. M. Abumandour, "Numerical study of slip effect of unsteady MHD pulsatile flow through porous medium in an artery using generalized differential quadrature method (comparative study)," World Journal of Engineering and Technology, vol. 2, no. 2, pp. 131-148, 2014.

[32] S. Akram, "Effects of slip and heat transfer on a peristaltic flow of a Carreau fluid in a vertical asymmetric channel," Computational Mathematics and Mathematical Physics, vol. 54, no. 12, pp. 1886-1902, 2014.

[33] A. Safia, S. Nadeem, and A. Hussin, "Influence of induced magnetic field and partial slip on the peristaltic flow of a couple stress fluid in an asymmetric channel," Iranian Journal of Chemistry and Chemical Engineering, vol. 33, pp. 43-52, 2014. 
[34] Y. C. Fung and C. S. Yih, "Peristaltic transport," ASME Journal of Applied Mechanics, vol. 33, pp. 669-675, 1968.

[35] D. A. Drew, "Stability of a stokes' layer of a dusty gas," Physics of Fluids, vol. 22, no. 11, pp. 2081-2084, 1979.

[36] G. K. Batchelor, "Transport properties of two-phase materials with random structure," Annual Review of Fluid Mechanics, vol. 6, pp. 227-255, 1974.

[37] G. K. Batchelor, "Brownian diffusion of particles with hydrodynamic interaction," Journal of Fluid Mechanics, vol. 74, no. 1, pp. 1-29, 1976.

[38] C. K. W. Tam, “The drag on a cloud of spherical particles in low Reynolds number flow," Journal of Fluid Mechanics, vol. 38, no. 3, pp. 537-546, 1969.

[39] S. E. Charm and G. S. Kurland, Blood Flow and MicroCirculation, John Wiley, New York, NY, USA, 1974.

[40] A. H. Shapiro, M. Y. Jaffrin, and S. L. Weinberg, "Peristaltic pumping with long wavelengths at low Reynolds number," Journal of Fluid Mechanics, vol. 37, no. 4, pp. 799-825, 1969.

[41] F. C. Yin and Y. C. Fung, "Comparison of theory and experiment in peristaltic transport," Journal of Fluid Mechanics, vol. 47, no. 01, pp. 93-112, 1971. 

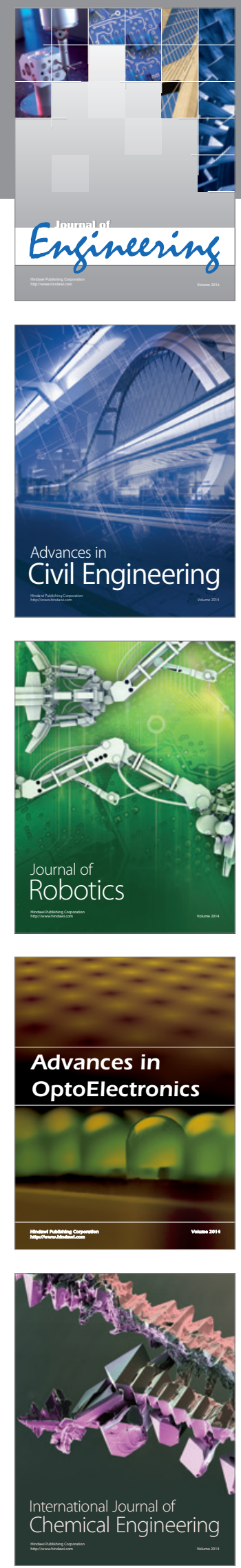

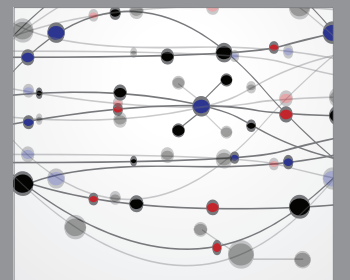

The Scientific World Journal
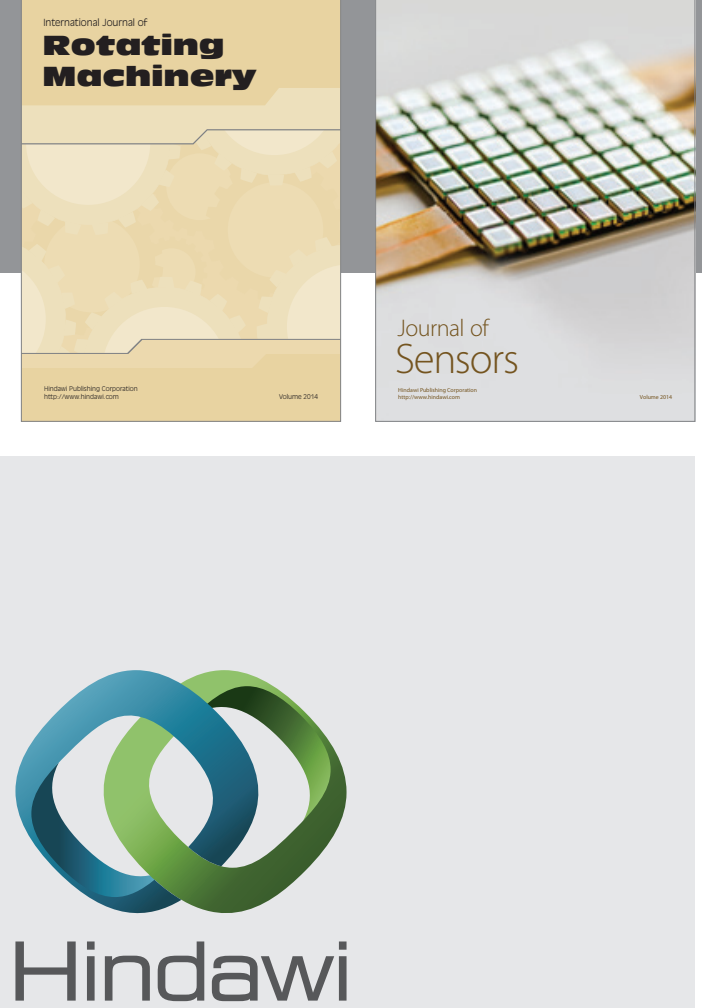

Submit your manuscripts at http://www.hindawi.com
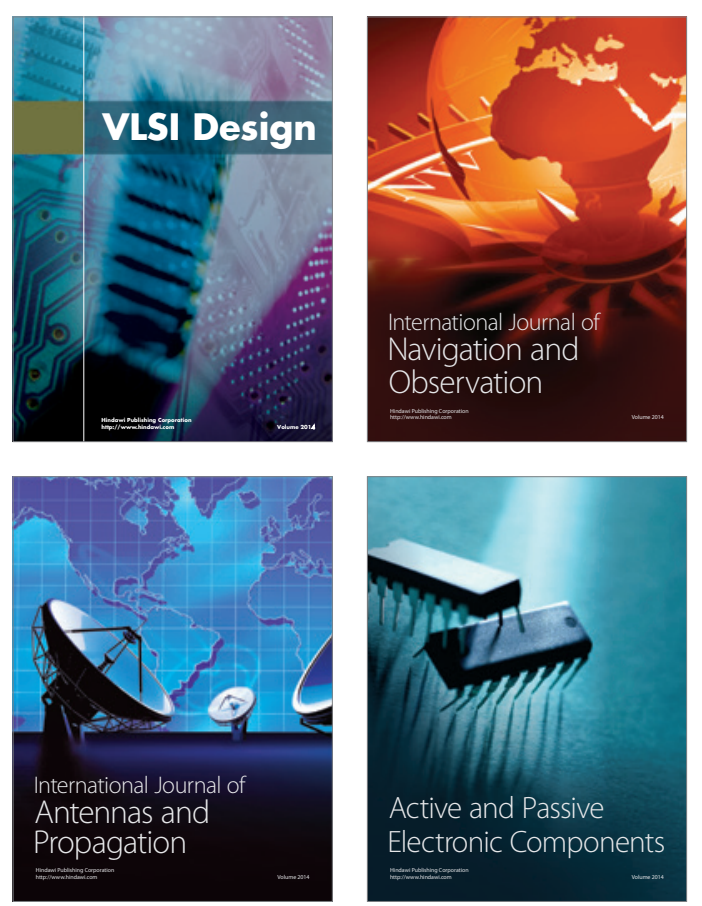
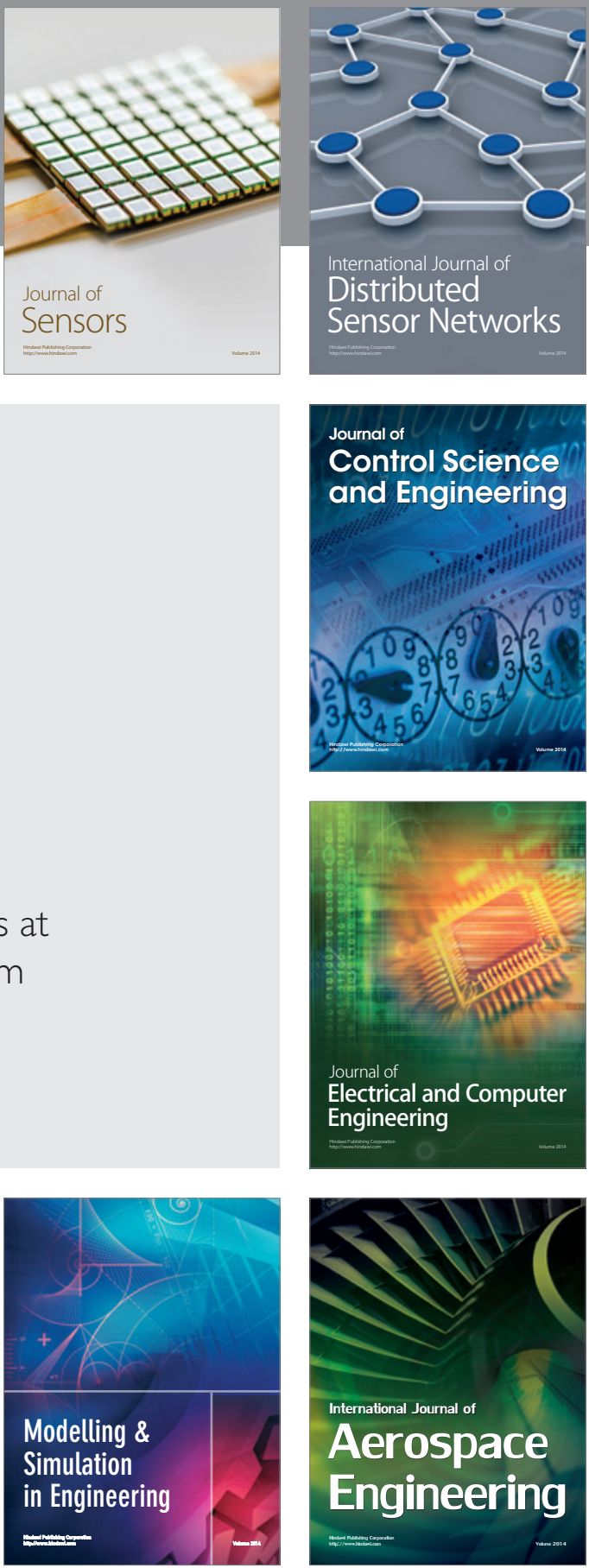

Journal of

Control Science

and Engineering
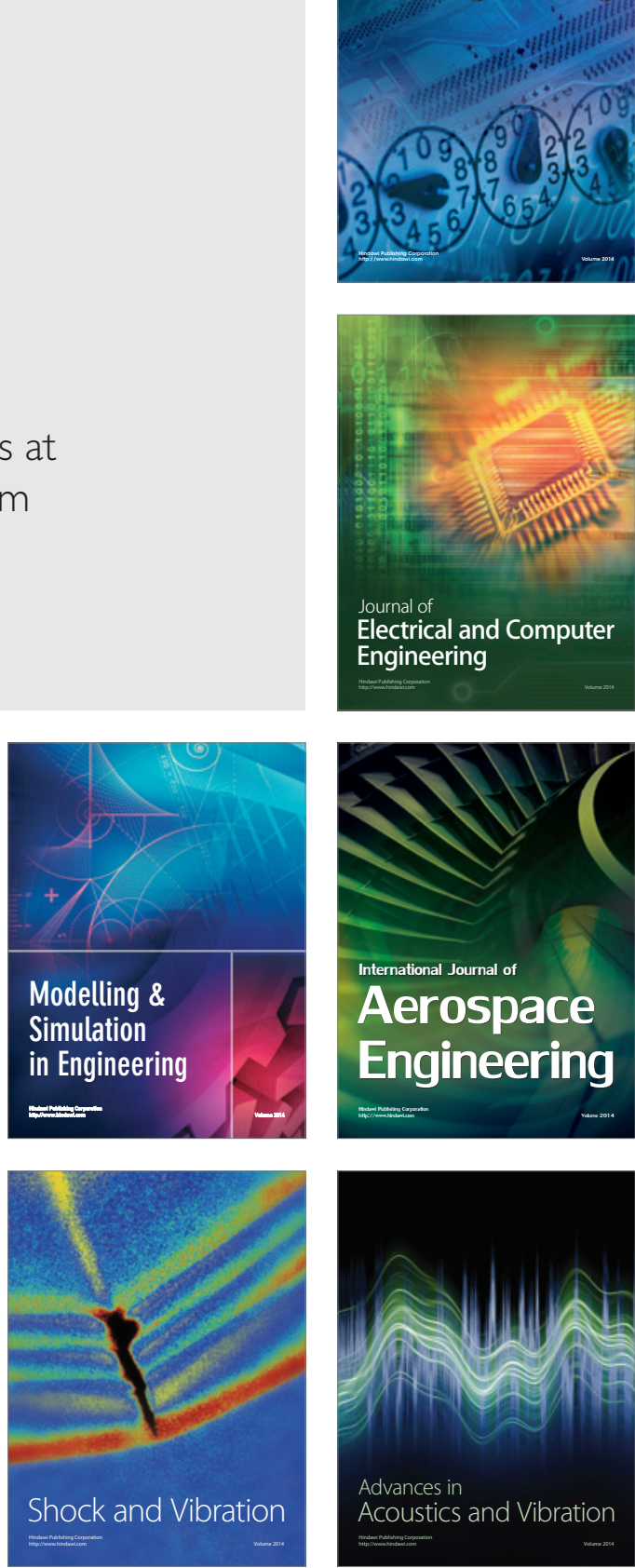